\title{
Adaptive Backstepping Controller for Uncertain Systems With Unknown Input Time-Delay. Application to SI Engines
}

\author{
Delphine Bresch-Pietri, Jonathan Chauvin, Nicolas Petit
}

\begin{abstract}
In this paper, we study the equilibrium regulation of potentially unstable linear systems with an unknown input time-delay and unknown parameters in the plant. We extend recent results from the literature where such systems are treated using a backstepping approach applied to a distributed parameters system representation of the delay. We develop a local result, robust to delay errors and apply it for the control of the Air-Fuel Ratio in Spark Ignition engines. A proof of convergence is established for this particular example. Experimental results stress the relevance of the proposed control algorithm.
\end{abstract}

\section{INTRODUCTION}

As is well established, see e.g. [14], time-delays represent a challenge in control design. This is true in various fields of engineering: chemical processes, biological reactors, communication networks and in general systems incorporating some transport phenomena. Further, actuators and sensors involved in feedback loops usually introduce additional sources of delays.

In this article, we focus on one such problem: the control of the Air-Fuel Ratio (AFR) in Spark-Ignition (SI) engines using the injection system. For a few decades, in a context of constantly increasing requirements in terms of pollutant emissions reduction and fuel saving, accurate control of the AFR has gradually become an issue of significant importance ${ }^{1}$. As every existing closed-loop control solutions for this problem, the strategy we propose relies on a signal given by an Oxygen Sensor located in the exhaust line. The transport of gas in the exhaust line and the sensor response both contribute to the appearance of a delay.

In this application, the involved system parameters and the value of the delay itself vary across the engine operating range. This situation can be handled using carefully gainscheduled PID controllers, which require substantial experimental tuning efforts, especially to handle transients. This is why, recently, numerous techniques have been proposed to provide alternatives to this relatively tedious solution (neural networks ([1], [3]), adaptive methodologies, Kalman Filters

D. Bresch-Pietri (corresponding author) is a $\mathrm{PhD}$ Candidate in Mathematics and Control at MINES ParisTech, 60, Bd St-Michel, 75272 Paris, Cedex 06, France. Email : delphine.bresch-pietri@mines-paristech.fr

J. Chauvin is with the Department of Engine Control in IFP Energies Nouvelles, 1 et 4 Avenue du Bois Préau, 92852 Rueil Malmaison, France

N.Petit is with the Centre Automatique et Systèmes, Unité Mathématiques et Systèmes at MINES ParisTech, 60 Bd St Michel, 75272 Paris, France

${ }^{1}$ The Three-Way Catalysts (TWC) efficiency strongly depends on the regulation of the AFR. For this reason, conventional PID-type AFR controllers based on mean value models and tuned e.g. with the Ziegler-Nichols method (see e.g. [16] for details) are often regarded as a bottleneck and calibration time consuming.

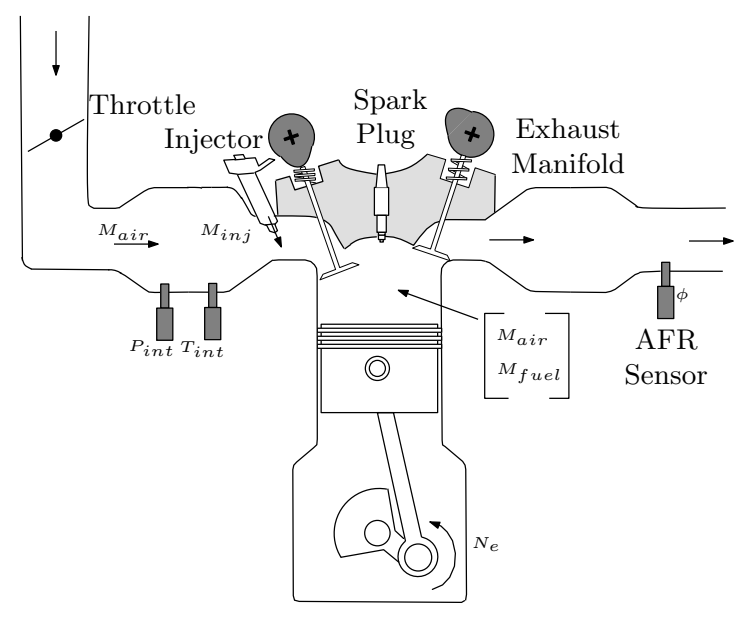

Fig. 1. Scheme of a Spark Ignition engine equipped with indirect injection, from the throttle down to the phi Sensor.

[12], model predictive control [8] among others). Nevertheless, these methods do not explicitly take into account the delay which is the root of practical difficulties.

Recently (see [5], [6], [11]), a new approach has been proposed to treat such systems, even in the presence of uncertain parameters in the plant and uncertainty in the delay value. This technique is a form of backstepping boundary control for partial differential equations (PDEs), modeling the actuator delay as a transport process. In this framework, results on delay-adaptive control for input-delay systems have been presented in [5], under the assumption that, except for the delay, all the parameters are perfectly known. This limitation has been removed in [6]. In this paper, we propose several changes in the adaptation laws to improve the applicability of the method.

As in most of the research on control of plants with a long actuator delay (especially potentially unstable ones), the approach uses a predictor-like feedback, based on the Arstein model reduction (see [4]). This reduction strategy has been shown to overcome some of the inherent problems of the conventional Smith predictor method [13].

Compared to previous results from the literature ([6], [10], [11]), the proposed technique does not assume that the full actuator state (i.e. past values of the input) is known over an interval of length equal to the delay. This lack of information does not prevent us from stabilizing the system, but this is done at the expense of global asymptotic stability which becomes only local (i.e. we require that the delay and the initial parameters estimates are sufficiently close to true values). 
This new control strategy, presented in Section III in (14)(17), and its application to a problem of engineering interest, are the main contributions of this article. A limitation of our work is that the trajectory tracking is here reduced to the tracking of a constant set-point. This assumption, which is not a problem for the application treated here, could be relaxed in future works.

The paper is organized as follows. In Section II, we describe the general framework of the problem under consideration, before presenting in Section III the general adaptive control strategy we propose. Then, in Section IV, we present the AFR control problem and apply the control strategy. For tutorial reasons, asymptotic convergence is only proven in this (relatively) simple case. Finally, experimental results are exposed in Section IV. The obtained performance is representative of state-of-the-art (i.e. it favorably compares to very carefully tuned PID). This shows the merit of our approach, which we now wish to apply and study on multidimensional systems.

\section{Problem Statement and Assumptions}

We consider the following potentially open-loop unstable delay system

$$
\dot{X}(t)=A(\theta) X(t)+B(\theta) U(t-D),
$$

where $X \in \mathbb{R}^{n}$ is the state and $U$ is a scalar input. $D>0$ is an unknown (potentially long) constant delay and we assume that the system matrix $A(\theta)$ and the input vector $B(\theta)$ are linearly parametrized under the form

$$
A(\theta)=A_{0}+\sum_{i=1}^{p} A_{i} \theta_{i} \quad \text { and } \quad B(\theta)=B_{0}+\sum_{i=1}^{p} B_{i} \theta_{i}
$$

where $\theta$ is an unknown constant vector of parameters belonging to a convex set $\Pi \subset \mathbb{R}^{p}$. Following [6], several assumptions are made.

Assumption 1: The set $\Pi$ is known and bounded. An upper bound $\bar{D}$ and a lower bound $\underline{D}>0$ of the delay $\mathrm{D}$ are known.

Assumption 2: For a given set point $X^{r} \in \mathbb{R}^{n}$, we assume that there exists a known function $U^{r}(\theta)$ continuously differentiable in the parameter $\theta \in \Pi$ such that $\left(X^{r}, U^{r}\right)$ satisfy

$$
0=A(\theta) X^{r}+B(\theta) U^{r}(\theta)
$$

Assumption 3: We assume that the pair $(A(\theta), B(\theta))$ is controllable for each $\theta \in \Pi$ and that there exists a triple of vector/matrix functions $(K(\theta), P(\theta), Q(\theta))$ such that

i) $P(\theta)$ and $Q(\theta)$ are positive definite and symmetric for each value of $\theta \in \Pi$

ii) the following Lyapunov equation is satisfied for $\theta \in \Pi$

$$
P(\theta)(A+B K)(\theta)+(A+B K)(\theta)^{T} P(\theta)=-Q(\theta)
$$

iii) $(K, P) \in C^{1}(\Pi)^{2}$ and $Q \in C^{0}(\Pi)$.

Assumption 4: The following quantities are well-defined

$$
\begin{aligned}
& \underline{\lambda}=\inf _{\theta \in \Pi} \min \left\{\lambda_{\min }(P(\theta)), \lambda_{\min }(Q(\theta))\right\} \\
& \bar{\lambda}=\sup _{\theta \in \Pi} \lambda_{\max }(P(\theta))
\end{aligned}
$$

The control objective is to have system (1) track the set-point $X^{r}$ through a full-state feedback. Among these assumptions, only one is truly restrictive: Assumption 3 requires the equivalent delay-free form of the system (1) to be controllable. This is a reasonable assumption to guarantee the possibility of regulation about the constant reference $X^{r}$. Assumptions 1 and 2 are formulated for well-posedness of the problem. Finally, Assumption 4 is formulated for Lyapunov design purposes only.

As a final remark, we wish to stress that the considered reference $U^{r}$ does not depend on time or delay, because the reference $X^{r}$ is constant. This point is crucial in the control design.

\section{CONTROL DESIGN}

We start our analysis by introducing the distributed input $u(x, t)=U(t+D(x-1)), x \in[0,1]$. The plant (1) can be represented under the form

$$
\begin{aligned}
\dot{X}(t) & =A(\theta) X(t)+B(\theta) u(0, t) \\
D u_{t}(x, t) & =u_{x}(x, t) \\
u(1, t) & =U(t),
\end{aligned}
$$

where the delay is accounted for by the transport equation whose speed of propagation is $1 / D$. Unfortunately, because this speed is uncertain, even if the applied input $U(t)$ is fully known, one cannot deduce the value of $u(x, t)$ for each $x \in[0,1]$ from it. Therefore, we introduce an estimate of the actuator state $\hat{u}(x, t)=U(t+\hat{D}(x-1))$, using the delay estimate $\hat{D}$. As will appear, no particular effort is made to update $\hat{D}$. For now, it is kept constant ${ }^{2}$.

Consider the following error variables

$$
\begin{aligned}
\tilde{X}(t) & =X(t)-X^{r} \\
\tilde{U}(t) & =U(t)-U^{r}(\hat{\theta}) \\
e(x, t) & =u(x, t)-u^{r}(\hat{\theta}) \\
\hat{e}(x, t) & =\hat{u}(x, t)-u^{r}(\hat{\theta}) \\
\tilde{e}(x, t) & =e(x, t)-\hat{e}(x, t)=u(x, t)-\hat{u}(x, t)
\end{aligned}
$$

In details, (8) quantifies the tracking error of the reference $X^{r},(12)$ is the estimation error of the distributed input, while (9)-(11) account for the estimation error of the unknown parameters $\theta$ and the output tracking error.

The infinite-dimensional state of the system and the actuator estimation error are fully described by the vector $(\tilde{X}, e, \hat{e})$.

When both the delay and the parameters of the system are known, the following controller (see [4]) achieves asymptotic stabilization of system (1) toward 0

$$
U(t)=K X_{P}(t+D)=K\left(e^{A D} X(t)+\int_{t-D}^{t} e^{A(t-s)} B U(s) d s\right)
$$

This controller can be viewed as a delay-version of the delay-free controller $U(t)=K X(t)$, where $X_{P}(t+D)$ should be understood as a D-units of time ahead prediction of the system state, starting from $X(t)$ as initial condition,

\footnotetext{
2 the design of a suitable delay update law is a direction of future works.
} 
and driven by the control history over the $D$-units of time window. This control has been interpreted in [10] as the result of a backstepping transformation of the transport partial differential equation (PDE) (7b) ${ }^{3}$. We follow this analysis, and the one pursued in [5] and [6]. We employ here the control law

$$
\begin{aligned}
U(t)= & U^{r}(\hat{\theta})+K(\hat{\theta}) \tilde{X}_{P}(t+\hat{D}) \\
= & U^{r}(\hat{\theta})-K(\hat{\theta}) X^{r}+K(\hat{\theta})\left[e^{A(\hat{\theta}) \hat{D}} X(t)\right. \\
& \left.+\hat{D}(t) \int_{0}^{1} e^{A(\hat{\theta}) \hat{D}(1-y)} B(\hat{\theta}) \hat{u}(y, t) d y\right],
\end{aligned}
$$

based on the certainty equivalence principle. The delay estimate $\hat{D}$ is kept constant and the update law $\hat{\theta}$ is chosen as

$$
\begin{aligned}
\dot{\hat{\theta}}(t) & =\gamma \operatorname{Proj}_{\Pi}\left(\zeta_{\theta}\right) \\
\zeta_{\theta, i} & =\left(\frac{\tilde{X}(t)^{T} P(\hat{\theta})}{b_{2}}-\hat{D} K(\hat{\theta}) \int_{0}^{1}(1+x)[\hat{w}(x, t)\right. \\
& \left.\left.+A(\hat{\theta}) \hat{D}_{\hat{w}}(x, t)\right] e^{A(\hat{\theta}) \hat{D} x} d x\right)\left(A_{i} X(t)+B_{i} u^{r}(\hat{\theta})\right)
\end{aligned}
$$

with $\gamma>0,1 \leq i \leq p$ and where the transformed estimate state of the actuator satisfies the following Volterra integral equation of the second kind

$$
\begin{aligned}
\hat{w}(x, t)= & \hat{e}(x, t)-\hat{D} \int_{0}^{x} K(\hat{\theta}) e^{A(\hat{\theta}) \hat{D}(x-y)} B(\hat{\theta}) \hat{e}(y, t) d y \\
& -K(\hat{\theta}) e^{A(\hat{\theta}) \hat{D} x} \tilde{X}(t)
\end{aligned}
$$

In (16), the matrix $P$ stands from Assumption 3, the constant $b_{2}$ is chosen such that $b_{2} \geq 8 \sup _{\theta \in \Pi}|P B|^{2} / \underline{\lambda}$, and $\operatorname{Proj}_{\Pi}$ is the standard projector operator onto the convex set $\Pi$ (see [6] for its formal definition).

From a comparison of (14)-(17) to the corresponding equations given in [6], one can observe that the main difference consists in the particular usage of the estimate actuator state $\hat{u}$ and the tracking error $\hat{e}$ (instead of the true values $u$ and $e$ ) respectively in the control (14) and the transformation of the actuator (17). A consequence appears in the expression of the update law (15)-(16), which involves a $H_{1}$-norm of the transformed state of the actuator and the reference distributed control.

Finally, denoting $\|$.$\| the spatial L^{2}$-norm, we define the following functional, which evaluates the system state

$$
\Gamma(t)=|\tilde{X}(t)|^{2}+\|e(t)\|^{2}+\|\hat{e}(t)\|^{2}+\left\|\hat{e}_{x}(t)\right\|^{2}+|\tilde{\theta}(t)|^{2}
$$

Comparing this definition to the one given in [6], we observe that both the norm of the estimate $\hat{e}$ and the norm of its spatial derivative have been added. An equivalent functional $V$ (defined below in (21)) will serve in the Lyapunov analysis.

Considering the closed-loop system consisting of (7a)(7c), the control law (14) and the update law defined through (15)-(16), we claim that there exists $\delta^{*}>0$ and $\gamma^{*}>0$ such that for any $|\tilde{D}|=|D-\hat{D}|<\delta^{*}$, i.e. for any constant

\footnotetext{
${ }^{3}$ this transformation is made to convert the plant (7a)-(7c) into the target system $\dot{X}(t)=(A+B K)(\theta) X(t)+B(\theta) w(0, t)$

$D w_{t}(x, t)=w_{x}(x, t)$ with the boundary condition $w(1, t)=0$.
}

$\hat{D} \in\left(D-\delta^{*}, D+\delta^{*}\right)$, there exist positive constants $R$ and $\rho$ such that, if the initial state $\left(\tilde{X}(0), e_{0}(),. \hat{e}_{0}(),. \tilde{\theta}(0)\right)$ is such that $\Gamma(0)<\rho$ and if $\gamma \in\left(0, \gamma^{*}\right)$, then

$$
\begin{gathered}
\Gamma(t) \leq R \Gamma(0), \\
\lim _{t \rightarrow \infty} \tilde{X}(t)=0, \quad \lim _{t \rightarrow \infty} \tilde{U}(t)=0
\end{gathered}
$$

The design of the update law (15)-(16) is directly based on a Lyapunov analysis using the following functional

$$
\begin{aligned}
V(t)= & \tilde{X}(t)^{T} P(\hat{\theta}) \tilde{X}(t)+b_{1} D \int_{0}^{1}(1+x) \tilde{e}(x, t)^{2} d x \\
& +b_{2} \hat{D}\left[\int_{0}^{1}(1+x) \hat{w}(x, t)^{2} d x\right. \\
& \left.+\int_{0}^{1}(1+x) \hat{w}_{x}(x, t)^{2} d x\right]+\frac{b_{2}}{\gamma}|\tilde{\theta}(t)|^{2},
\end{aligned}
$$

where $b_{1}$ and $b_{2}$ are strictly positive constants. The factor $(1+x)$ under the integral (also present in (16)) is handy in the Lyapunov analysis (through integrations by parts). The variables $(\tilde{X}, \tilde{e}, \hat{w})$ are equivalent to the original ones $(\tilde{X}, e, \hat{e})$ (via (17)) and reveal helpful in the analysis.

\section{Application to SPARK-IGNition Engines : AN Air/FuEl RATIO CONTROLlER}

In this section, we apply the general control strategy presented in the previous section to the problem of AFR control for a SI engine. We prove its convergence in this particular case. Most elements of proof can be generalized to multi-variable cases.

\section{A. Dynamics of the Air/Fuel Ratio}

Classically, in SI engines, the Air-Fuel Ratio is defined as the ratio between the air mass $M_{\text {air }}$ and the fuel mass $M_{\text {fuel }}$ filling the cylinder at each stroke. Here, we use the normalized inverse of the AFR

$$
\phi=\frac{M_{\text {fuel }}}{M_{\text {air }}} /\left(\frac{M_{\text {fuel }}}{M_{\text {air }}}\right)_{S}
$$

where $\left(M_{\text {fuel }} / M_{\text {air }}\right)_{S}$ is the stoichiometric Fuel/Air Ratio value. To maximize the efficiency of exhaust gases aftertreatment devices, $\phi$ has to be maintained as close as possible to the unity. In open-loop, the injected fuel mass is directly computed from the estimated value of the in-cylinder air mass, i.e. $M_{\text {inj }}=\phi_{S} M_{\text {air }}$.

To accurately control the AFR, in the presence of disturbances, a closed-loop strategy is considered. It uses the signal of an oxygen sensor located in the exhaust line, which is embedded in all commercial engines (see Fig.1). As pointed out in numerous studies (e.g. [15]), the dynamics of the sensor can be approximated by a low-pass transfer function, driven by a delayed input signal. In practice,

$$
\begin{aligned}
\tau_{\phi} \dot{\phi}(t) & =-\phi(t)+\alpha U(t-D) \\
\tau_{\phi} & =\tau_{\phi}\left(Q_{\text {air }}\right) \\
D & =D\left(N_{e}\right)
\end{aligned}
$$

where $U$ is related to the injected fuel mass $M_{i n j}$ through known relations, $N_{e}$ is the engine speed, and $Q_{a i r}$ is the 
aspirated air flow ${ }^{4}$. Here, we detail the involved parameters and their variation with the operating point in the $\left(N_{e}, Q_{\text {air }}\right)$ map.

1) The delay $D: D$ accounts both for the fact that the sensor is not located directly in the vicinity of the exhaust valve and for the dead time of the AFR sensor. It is usually assumed that $D$ solely depends on the engine speed, which is not totally true: $D$ is not well known. Nevertheless, some bounds for it can be established: $D \in[\underline{D}, \bar{D}]=[0.1 s, 0.6 s]$.

2) The time constant $\tau_{\phi}$ : we assume that we have an accurate knowledge of $\tau_{\phi}$, which can be readily identified as a function of the aspirated air flow. This constant represents the time needed to fill the porous coating layers protecting the electrodes of the sensor.

3) The gain $\alpha$ : this quantity aggregates various factors, mainly the errors in the estimation of the in-cylinder air mass and the effects of the electronic injection devices lag. This term cannot be easily measured and, furthermore, varies over time due to devices aging among other causes. Therefore, this quantity is uncertain, even if, as can be observed, its variability is relatively small $(\alpha \in[\underline{\alpha}, \bar{\alpha}]=[0.75 ; 1.25])$ and low-frequency.

\section{B. General strategy}

To sum up the above discussion, for a given operating point $\left(N_{e}, Q_{\text {air }}\right)$, we wish to stabilize $\phi$ around the value $\phi^{r}$, which is usually equal to the unity ${ }^{5}$. We control this value via the injected fuel mass, adapting the estimate $\hat{\alpha}$ of the unknown parameter $\alpha$ and using the estimated value $\hat{D}=D\left(N_{e}\right)$. In this context, all the parameters are constant.

\section{Proof of convergence of $\phi$}

Using the notations of Section II, we define $X=\phi, \theta=$ $\alpha, A=A_{0}=1 / \tau_{\phi}$ and $B(\alpha)=B_{1} \alpha=\alpha / \tau_{\phi}$. We note $\phi^{r}$ the inverse AFR set-point. It is straightforward to satisfy Assumption 2 with the reference control $U^{r}=\phi^{r} / \alpha$. The system (23) is obviously controllable; we arbitrarily set the gain $K=-1, P=\tau_{\phi}$ and, consequently, $Q(\alpha)=2(1+\alpha)$. Finally, because $\alpha$ is bounded and strictly positive, Assumption 4 is satisfied. Then the quantities defined through Section III are

$$
\begin{aligned}
\tilde{\phi}(t) & =\phi(t)-\phi^{r} \\
\tilde{U}(t) & =U(t)-\phi^{r} / \hat{\alpha}(t) \\
\tilde{e}(x, t) & =u(x, t)-\hat{u}(x, t) \\
\hat{e}(x, t) & =\hat{u}(x, t)-\phi^{r} / \hat{\alpha}(t)
\end{aligned}
$$

We claim that the control law

$$
\begin{aligned}
U(t)= & \frac{\phi^{r}}{\hat{\alpha}(t)}+\phi^{r}-e^{-\hat{D} / \tau_{\phi}} \phi(t) \\
& -\hat{D} \frac{\hat{\alpha}(t)}{\tau_{\phi}} \int_{0}^{1} e^{-\hat{D}(1-y) / \tau_{\phi}} \hat{u}(y, t) d y
\end{aligned}
$$

\footnotetext{
${ }^{4}$ In details, the quantity measured by the oxygen sensor is the exhaust equivalent ratio $\phi_{e q}=M_{t o t} / M_{b g}$ (where $M_{t o t}$ represents the exhaust gas mass and $M_{b g}$ the exhaust burned gases mass), which is directly related to the intake Fuel-Air ratio.

${ }^{5}$ even if, for some operating point, it is useful to define $\phi^{r}>1$ in order to decrease the exhaust temperature.
}

achieves tracking of the reference $\phi^{r}$, jointly with a constant and sufficiently accurate estimate $\hat{D}$ of $D$ and the update law 6

$$
\begin{aligned}
& \dot{\hat{\alpha}}(t)= \gamma \frac{\phi^{r}}{\hat{\alpha}(t)} h(t) \\
& h(t)=\frac{\tilde{\phi}(t)}{b_{2}}+\frac{\hat{D}}{\tau_{\phi}} \int_{0}^{1}(1+x)\left[\hat{w}(x, t)-\frac{\hat{D}}{\tau_{\phi}} \hat{w}_{x}(x, t)\right] \\
& \times e^{-\hat{D} x / \tau_{\phi}} d x
\end{aligned}
$$

when $\hat{\alpha}$ is in its definition field, with $b_{2} \geq \frac{4 \bar{\alpha}^{2}}{1+\alpha}, \gamma>0$ sufficiently small and the transformed state of the actuator

$$
\begin{aligned}
\hat{w}(x, t)= & \hat{e}(x, t)+\hat{D} \frac{\hat{\alpha}(t)}{\tau_{\phi}} \int_{0}^{x} e^{-\hat{D}(x-y) / \tau_{\phi}} \hat{e}(y, t) d y \\
& +e^{-\hat{D} x / \tau_{\phi}} \tilde{\phi}(t) .
\end{aligned}
$$

When $\hat{\alpha}$ reaches the boundaries of $[\underline{\alpha} ; \bar{\alpha}]$, it is saturated using the projection operator introduced in (15).

To prove this point, and before working with the Lyapunov functional defined in (21), we consider the mapping (33) to obtain the following transformed system

$$
\begin{aligned}
\tau_{\phi} \dot{\tilde{\phi}}(t)= & -(1+\hat{\alpha}(t)) \tilde{\phi}(t)+\hat{\alpha}(t) \hat{w}(0, t) \\
& +\tilde{\alpha}(t) u(0, t)+\hat{\alpha}(t) \tilde{e}(0, t) \\
D \tilde{e}_{t}(x, t)= & \tilde{e}_{x}(x, t)-\tilde{D} f(x, t) \\
\tilde{e}(1, t)= & 0 \\
\hat{D} \hat{w}_{t}(x, t)= & \hat{w}_{x}(x, t)+\hat{D} \dot{\hat{\alpha}}(t) g(x, t) \\
& +\frac{\hat{D}}{\tau_{\phi}} e^{-\hat{D} x / \tau_{\phi}}(\tilde{\alpha}(t) u(0, t)+\hat{\alpha}(t) \tilde{e}(0, t)) \\
\hat{w}(1, t)= & 0,
\end{aligned}
$$

where $f$ and $g$ are defined in Appendix $\mathrm{A}^{7}$. As our Lyapunov analysis involves an $H_{1}$ norm of $\hat{w}$ (due to the expression of $f(x, t)$ ), we also need the governing equations of the $\hat{w}_{x^{-}}$ system. These are

$$
\begin{aligned}
& \hat{D} \hat{w}_{x t}(x, t)=\hat{w}_{x x}(x, t)+\hat{D} \dot{\hat{\alpha}}(t) g_{x}(x, t) \\
& -\frac{\hat{D}^{2}}{\tau_{\phi}^{2}} e^{-\hat{D} x / \tau_{\phi}}(\tilde{\alpha}(t) u(0, t)+\hat{\alpha}(t) \tilde{e}(0, t)) \\
& \hat{w}_{x}(1, t)=-\hat{D} \dot{\hat{\alpha}}(t) g(1, t) \\
& -\frac{\hat{D}}{\tau_{\phi}} e^{-\hat{D} / \tau_{\phi}}(\tilde{\alpha}(t) u(0, t)+\hat{\alpha}(t) \tilde{e}(0, t))
\end{aligned}
$$

One could observe that the estimation error of the unknown parameter appears both in the error model (34) and in the subsystem (36)-(37), which is reflected in the update law (31)-(32).

In the time-derivative of $V$, one can create dominant negative terms, through some judicious integrations by parts using the zero boundary conditions of the two systems (35)(36). To prove that this derivative can be made negative

\footnotetext{
${ }^{6}$ one can observe that, in the equivalent non-delayed problem $(D=\hat{D}=0)$ the control and update laws mentioned in (14)-(16) are the one obtained by an usual adaptive method : $U(t)=\phi^{r} / \hat{\alpha}(t)-\tilde{\phi}(t)$ and $\dot{\hat{\alpha}}(t)=\phi^{r} \tilde{\phi}(t) / \hat{\alpha}(t)$.

${ }^{7}$ these functions could be expressed in simpler forms than the one given in Appendix A (e.g. $f(x, t)=\hat{e}(x, t) / \hat{D})$, but, for Lyapunov analysis purposes, we need to express them with the variables $\left(\tilde{\phi}, \tilde{e}, \hat{w}, \hat{w}_{x}\right)$.
} 
semidefinite, we bound most of the terms resulting from the differentiation of $V$, except the estimation errors ones. In fact, these terms are factors of a non-vanishing term, $u(0, t)$. Yet, the actuator state is not known, and, therefore, it is not possible to cancel these terms. The best that can be done is to create a vanishing term, namely $e(0, t)$ here.

a) Lyapunov Analysis: The Lyapunov functional (21) can here be written, with $b_{1}>0$,

$$
\begin{aligned}
& V(t)=\tau_{\phi} \tilde{\phi}(t)^{2}+\frac{b_{2}}{\gamma} \tilde{\alpha}(t)^{2}+b_{1} D \int_{0}^{1}(1+x) \tilde{e}(x, t)^{2} d x \\
& +b_{2} \hat{D}\left(\int_{0}^{1}(1+x) \hat{w}(x, t)^{2} d x+\int_{0}^{1}(1+x) \hat{w}_{x}(x, t)^{2} d x\right)
\end{aligned}
$$

Using integrations by parts and the boundary conditions (35) and (36), its time derivative is

$$
\begin{aligned}
& \dot{V}(t)=-2(1+\hat{\alpha}(t)) \tilde{\phi}(t)^{2}+2 \hat{\alpha}(t) \tilde{\phi}(t)(\hat{w}(0, t)+\tilde{e}(0, t)) \\
& +2 \tilde{\alpha}(t) \tilde{\phi}(t) u(0, t)-\frac{2 b_{2}}{\gamma} \tilde{\alpha}(t) \dot{\hat{\alpha}}(t)+b_{1}\left(-\tilde{e}(0, t)-\|\tilde{e}(t)\|^{2}\right. \\
& \left.-2 \tilde{D} \int_{0}^{1}(1+x) \tilde{e}(x, t) f(x, t) d x\right)+b_{2}\left(-\hat{w}(0, t)-\|\hat{w}(t)\|^{2}\right. \\
& +2 \hat{D} \dot{\hat{\alpha}}(t) \int_{0}^{1}(1+x) \hat{w}(x, t) g(x, t) d x+\frac{2 \hat{D}}{\tau_{\phi}}(\tilde{\alpha}(t) u(0, t) \\
& \left.+\hat{\alpha}(t) \tilde{e}(0, t)) \int_{0}^{1}(1+x) \hat{w}(x, t) e^{-\hat{D} x / \tau_{\phi}} d x\right)+b_{2}\left(2 \hat{w}_{x}(1, t)^{2}\right. \\
& -\hat{w}_{x}(0, t)^{2}-\left\|\hat{w}_{x}(t)\right\|^{2}+2 \hat{D} \dot{\hat{\alpha}}(t) \int_{0}^{1}(1+x) \hat{w}_{x}(x, t) g_{x}(x, t) d x \\
& \left.-\frac{2 \hat{D}^{2}}{\tau_{\phi}^{2}}(\tilde{\alpha}(t) u(0, t)+\hat{\alpha} \tilde{e}(0, t)) \int_{0}^{1}(1+x) \hat{w}_{x}(x, t) e^{-\hat{D} x / \tau_{\phi}} d x\right)
\end{aligned}
$$

Then, using the update law (31) and the fact that $b_{2} \geq 4 \frac{\bar{\alpha}^{2}}{1+\underline{\alpha}}$ jointly with Young's inequality [10], we obtain

$$
\begin{aligned}
& \dot{V}(t) \leq-(1+\hat{\alpha}(t)) \tilde{\phi}(t)^{2}-\left(b_{1}-\frac{b_{2}}{2}\right) \tilde{e}(0, t)^{2}-\frac{b_{2}}{2} \hat{w}(0, t)^{2} \\
& -b_{1}\|\tilde{e}(t)\|^{2}-b_{2}\|\hat{w}(t)\|^{2}-b_{2}\left\|\hat{w}_{x}(t)\right\|^{2} \\
& +2 b_{2}|\tilde{\alpha}(t)||h(t)|\left|u(0, t)-\frac{\phi^{r}}{\hat{\alpha}(t)}\right|+2 b_{2} \hat{w}_{x}(1, t)^{2} \\
& +2 b_{1}|\tilde{D}| \int_{0}^{1}(1+x)|\tilde{e}(x, t)||f(x, t)| d x \\
& +2 b_{2} \hat{D}|\dot{\hat{\alpha}}(t)| \int_{0}^{1}(1+x)|\hat{w}(x, t)||g(x, t)| d x \\
& +2 b_{2} \hat{D}|\dot{\hat{\alpha}}(t)| \int_{0}^{1}(1+x)\left|\hat{w}_{x}(x, t)\right|\left|g_{x}(x, t)\right| d x+\frac{2 b_{2} \hat{D} \hat{\alpha}(t)}{\tau_{\phi}} \\
& \times|\tilde{e}(0, t)| \int_{0}^{1}(1+x)\left[|\hat{w}(x, t)|+\frac{\hat{D}}{\tau_{\phi}}\left|\hat{w}_{x}(x, t)\right|\right] e^{-\hat{D} x / \tau_{\phi}} d x
\end{aligned}
$$

With the inequalities given in Appendix B, it is easy to get

$$
\begin{aligned}
& \dot{V}(t) \leq-\frac{\tilde{\phi}(t)^{2}}{2}-\left(b_{1}-b_{2}\left(\frac{1}{2}+2 \bar{\alpha}\left(M_{1}+M_{2}+M_{3}\right)\right.\right. \\
& \left.\left.+2 M_{7}+2 M_{8}+M_{10}+4 \bar{\alpha}^{2} M_{11}\right)\right) \tilde{e}(0, t)^{2}-\frac{b_{2}}{2} \hat{w}(0, t)^{2}
\end{aligned}
$$

$$
\begin{aligned}
& -b_{1}\|\tilde{e}(t)\|^{2}-\frac{b_{2}}{2}\|\hat{w}(t)\|^{2}-\frac{b_{2}}{2}\left\|\hat{w}_{x}(t)\right\|^{2}+b_{2}|\tilde{\alpha}(t)|\left(M_{1}\right. \\
& \times\left(\tilde{\phi}(t)^{2}+\left\|\hat{w}_{x}(t)\right\|^{2}\right)+M_{2}\left(\tilde{\phi}(t)^{2}+\|\hat{w}(t)\|^{2}+\left\|\hat{w}_{x}(t)\right\|^{2}\right) \\
& \left.+M_{3}\left(\tilde{\phi}(t)^{2}+\left\|\hat{w}_{x}(t)\right\|^{2}\right)+2 M_{11} \bar{\alpha}\left(\tilde{\phi}(t)^{2}+\left\|\hat{w}_{x}(t)\right\|^{2}\right)\right) \\
& +|\tilde{D}| b_{1} M_{4}\left(\tilde{\phi}(t)^{2}+\|\tilde{e}(t)\|^{2}+\|\hat{w}(t)\|^{2}+\left\|\hat{w}_{x}(t)\right\|^{2}\right) \\
& +b_{2}|\dot{\hat{\alpha}}(t)|\left(M_{5}\left(\tilde{\phi}(t)^{2}+\|\hat{w}(t)\|^{2}+|\tilde{\phi}(t)|+\|\hat{w}(t)\|\right)\right. \\
& \left.+M_{6}\left(\tilde{\phi}(t)^{2}+\|\hat{w}(t)\|^{2}+\left\|\hat{w}_{x}(t)\right\|^{2}+|\tilde{\phi}(t)|+\left\|\hat{w}_{x}(t)\right\|\right)\right) \\
& +b_{2} M_{9} \dot{\hat{\alpha}}(t)^{2}\left(\|\hat{w}(t)\|^{2}+1\right)
\end{aligned}
$$

Choosing $b_{1}>b_{2}\left(1 / 2+2 \bar{\alpha}\left(M_{1}+M_{2}+M_{3}\right)+2 M_{7}+\right.$ $2 M_{8}+M_{10}+4 \bar{\alpha}^{2} M_{11}$ ), with inequality (58) and Young's inequality applied to the arising cubic terms, we obtain

$$
\begin{aligned}
& \dot{V}(t) \leq-\left(\eta-|\tilde{\alpha}(t)|\left(b_{2}\left(M_{1}+M_{2}+M_{3}\right)+2 b_{2} \bar{\alpha} M_{11}\right)\right. \\
& \left.-b_{2}\left(\frac{9}{2} \gamma\left[M_{5}+M_{6}\right] M_{12}+3 \gamma^{2} M_{9} M_{12}^{2}\right)-|\tilde{D}| b_{1} M_{4}\right) V_{0}(t) \\
& +3 b_{2} / 2 \times\left(\gamma\left[M_{5}+M_{6}\right] M_{12}+6 \gamma^{2} M_{9} M_{12}^{2}\right) V_{0}(t)^{2}
\end{aligned}
$$

where $\eta>0$ and

Then, the following bound of the parameter estimation error is employed

$$
|\tilde{\alpha}(t)| \leq \frac{\varepsilon}{2}+\frac{|\tilde{\alpha}(t)|^{2}}{2 \varepsilon} \leq \frac{\varepsilon}{2}+\frac{\gamma}{2 \varepsilon b_{2}}\left(V(t)-\eta_{1} V_{0}(t)\right),
$$

where $\eta_{1}=\min \left\{\tau_{\phi}, b_{1} \underline{D}, b_{2} \underline{D}\right\}$, which yields, with $m_{1}=$ $b_{2}\left(M_{1}+M_{2}+M_{3}\right)+2 b_{2} \bar{\alpha} M_{11}$ and $m_{2}=3 b_{2} / 2 \times\left(\gamma\left(M_{5}+\right.\right.$ $\left.\left.M_{6}\right) M_{12}+6 \gamma^{2} M_{9} M_{12}^{2}\right)$,

$$
\begin{aligned}
\dot{V}(t) & \leq-\left(\eta-|\tilde{D}| b_{1} M_{4}-m_{1}\left(\frac{\varepsilon}{2}+\frac{\gamma}{2 \varepsilon b_{2}} V(t)\right)-b_{2}\left(\frac { 9 } { 2 } \gamma \left[M_{5}\right.\right.\right. \\
& \left.\left.\left.+M_{6}\right] M_{12}+3 \gamma^{2} M_{9} M_{12}^{2}\right)\right) V_{0}(t)-\left(\frac{m_{1} \eta_{1} \gamma}{2 \varepsilon b_{2}}-m_{2}\right) V_{0}(t)^{2}
\end{aligned}
$$

Assuming that $|\tilde{D}|<\delta^{*}=\eta /\left(b_{1} M_{4}\right)$, defining $m_{3}(\gamma)=$ $b_{2}\left(9 / 2 \times \gamma\left[M_{5}+M_{6}\right] M_{12}+3 \gamma^{2} M_{9} M_{12}^{2}\right)$, choosing the parameter $\varepsilon$ and the gain $\gamma$ such that

$$
\begin{aligned}
& \gamma<\quad \gamma^{*}=\min \left\{\frac{\eta-|\tilde{D}| b_{1} M_{4}}{m_{3}(1)}, 1\right\} \\
& \varepsilon<\min \left\{\frac{2\left(\eta-|\tilde{D}| b_{1} M_{4}-m_{3}(\gamma)\right)}{m_{1}}, \frac{m_{1} \eta_{1} \gamma}{2 b_{2} m_{2}}\right\}
\end{aligned}
$$

and restricting the initial condition so that

$$
V(0) \leq \frac{2 \varepsilon b_{2}}{\gamma}\left(\eta-|\tilde{D}| b_{1} M_{4}-\frac{m_{1} \varepsilon}{2}-m_{3}(\gamma)\right),
$$

one obtains

$$
\dot{V}(t) \leq-\mu_{1}(t) V_{0}(t)-\mu_{2}(t) V_{0}(t)^{2},
$$

where $\mu_{1}$ and $\mu_{2}$ are positive. Therefore, the following stability property holds

$$
\forall t \geq 0, V(t) \leq V(0)
$$


b) Equivalence: In view of obtaining (19), we prove that the functional (18), which here takes the form

$$
\Gamma(t)=\tilde{\phi}(t)^{2}+\|e(x, t)\|^{2}+\|\hat{e}(x, t)\|^{2}+\left\|\hat{e}_{x}(x, t)\right\|^{2}+\tilde{\alpha}(t)^{2}
$$

is equivalent to $V$, i.e. that there exist constants $a>0$ and $b>0$ such that $a V(t) \leq \Gamma(t) \leq b V(t), \forall t \geq 0$. Considering (33) and the inverse transformation

$$
\begin{aligned}
\hat{e}(x, t)= & \hat{w}(x, t)-\frac{\hat{D} \hat{\alpha}(t)}{\tau_{\phi}} \int_{0}^{x} e^{-(1+\hat{\alpha}(t)) \hat{D}(x-y) / \tau_{\phi}} \hat{w}(y, t) d y \\
& -e^{-(1+\hat{\alpha}(t)) \hat{D} x / \tau_{\phi}} \tilde{\phi}(t),
\end{aligned}
$$

one obtains, using Cauchy-Schwartz's, Young's and Agmon's inequalities (see e.g. [10]),

$$
\begin{aligned}
\|\hat{e}(t)\|^{2} & \leq r_{1}\|\hat{w}(t)\|^{2}+r_{2} \tilde{\phi}(t)^{2} \\
\left\|\hat{e}_{x}(t)\right\|^{2} & \leq 4\left\|\hat{w}_{x}(t)\right\|^{2}+r_{3}\|\hat{w}(t)\|^{2}+r_{4} \tilde{\phi}(t)^{2} \\
\|\hat{w}(t)\|^{2} & \leq s_{1}\|\hat{e}(t)\|^{2}+s_{2} \tilde{\phi}(t)^{2} \\
\left\|\hat{w}_{x}(t)\right\|^{2} & \leq 4\left\|\hat{e}_{x}(t)\right\|^{2}+s_{3}\|\hat{e}(t)\|^{2}+s_{4} \tilde{\phi}(t)^{2}
\end{aligned}
$$

where $r_{1}, r_{2}, r_{3}, r_{4}, s_{1}, s_{2}, s_{3}$ and $s_{4}$ are positive constants. Then, it is easy to get

$$
\begin{aligned}
\Gamma(t) \leq & \tilde{\phi}(t)^{2}+2\|\tilde{e}(t)\|^{2}+3\left(r_{1}\|\hat{w}(t)\|^{2}+r_{2} \tilde{\phi}(t)^{2}\right) \\
& +4\left\|\hat{w}_{x}(t)\right\|^{2}+r_{3}\|\hat{w}(t)\|^{2}+r_{4} \tilde{\phi}(t)^{2}+\tilde{\alpha}(t)^{2} \\
\leq & \frac{\max \left\{1+3 r_{2}+r_{4}, 3 r_{1}+r_{3}, 4\right\}}{\min \left\{\tau_{\phi}, b_{1} \underline{D}, b_{2} \underline{D}, b_{2} / \gamma\right\}} V(t) \\
V(t) \leq & \max \left\{\tau_{\phi}, \frac{b_{2}}{\gamma}, 2 b_{1} \bar{D}, 2 b_{2} \bar{D}\right\} \\
& \times \max \left\{1+s_{2}+s_{4}, 2+s_{1}+s_{3}, 4\right\} \Gamma(t),
\end{aligned}
$$

which gives the equivalence between the two functionals. With this property, it is easy to deduce (19) from (41).

c) Tracking: Starting from the stability result (41), we now conclude using Barbalat's Lemma. From (38) and (41), it follows that $\tilde{\phi}(t),\|\tilde{e}(t)\|,\|\hat{w}(t)\|$ and $\left\|\hat{w}_{x}(t)\right\|$ are uniformly bounded. Then, with the inverse transformation of the actuator state (43), we obtain the uniform boundedness of $\|\hat{e}(t)\|$. Then, from (27) and (30), we get that the control error $\tilde{U}(t)$ is uniformly bounded for $t \geq 0$. Consequently, as the reference control $U^{r}(\hat{\alpha})$ is bounded on the set $[\underline{\alpha}, \bar{\alpha}]$, the control law $U(t)$ is uniformly bounded for $t \geq 0$. Then, $u(0, t)=U(t-D)$ and $e(0, t)=\tilde{U}(t-D)$ are uniformly bounded for $t \geq D$ and so are $\hat{e}(0, t)$ and $\tilde{e}(0, t)$ for $t \geq \max \{D, \hat{D}\}$. Besides,

$$
\begin{aligned}
& \tau_{\phi} \frac{d \tilde{\phi}(t)^{2}}{d t}=2 \tilde{\phi}(t)(-(1+\hat{\alpha}) \tilde{\phi}(t)+\hat{\alpha}(t) \hat{e}(0, t) \\
& +\tilde{\alpha}(t) u(0, t)+\hat{\alpha}(t) \tilde{e}(0, t))
\end{aligned}
$$

which yields the uniform boundedness of $d \tilde{\phi}(t)^{2} / d t$ for $t \geq$ $\max \{D, \hat{D}\}$. As $|\tilde{\phi}(t)|$ is square integrable from (40), we get, by Barbalat's Lemma, that $\tilde{\phi}(t) \rightarrow 0$ as $t \rightarrow \infty$.

Similarly,

$$
\begin{aligned}
\frac{d \tilde{U}(t)^{2}}{d t}= & 2 \tilde{U}(t)\left(-e^{-\hat{D} / \tau_{\phi}} \dot{\tilde{\phi}}(t)-\dot{\hat{\alpha}}(t) \frac{\hat{D}}{\tau_{\phi}} \int_{0}^{1} e^{-\hat{D}(1-y) / \tau_{\phi}}\right. \\
& \times\left(\frac{\phi^{r}}{\hat{\alpha}(t)}+\hat{e}(y, t)\right) d y+\frac{\hat{\alpha}(t)}{\tau_{\phi}}\left(\tilde{U}(t)-e^{-\hat{D} / \tau_{\phi}}\right.
\end{aligned}
$$

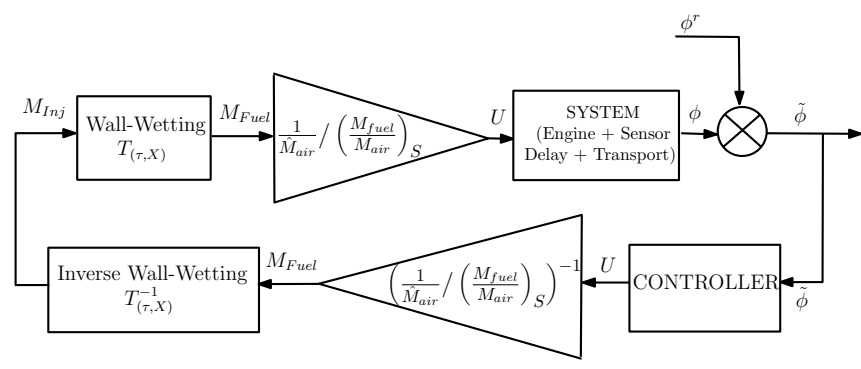

Fig. 2. Simplified block diagram of the strategy.

$$
\left.\left.\times \hat{e}(0, t)-\frac{\hat{D}}{\tau_{\phi}} \int_{0}^{1} e^{-\hat{D}(x-y) / \tau_{\phi}} \hat{e}(y, t) d y\right)\right)
$$

The signal $\dot{\hat{\alpha}}(t)$ is uniformly bounded for $t \geq 0$ according to (31). Then, $d \tilde{U}(t)^{2} / d t$ is bounded for $t \geq \max \{D, \hat{D}\}$. Besides, from (40), we get the square integrability of $\tilde{\phi}(t)$ and $\|\hat{w}(t)\|$. From (44), we obtain the square integrability of $\|\hat{e}(t)\|$. Then, $\tilde{U}(t)$ is square integrable, and we conclude, by Barbalat's Lemma, that $\tilde{U}(t) \rightarrow 0$ as $t \rightarrow \infty$.

\section{Transient control strategy}

The range of variation of the delay and the parameter $\alpha$ over the entire operating area is sufficiently small so that, at all times, the updated set-point lies in the vicinity of the current set-point. Consequently, the previously presented controller can be used in transient mode. No particular feedforward terms are needed.

Besides, it is possible to tune the transient behavior adjusting the gains $\gamma$ and $-K$ (set to 1 in the presented proof) to the operating point. This has not appeared necessary in the following experimental test.

\section{EXPERIMENTAL RESUltS}

We now present experimental results obtained using the proposed strategy. Constant gains $\gamma=1$ and $K=-1$ are used over the whole operating range.

\section{A. Experimental set-up}

All experimental results presented in this section have been obtained on a four-cylinder indirect injection $2 \mathrm{~L}$ engine (see [7] for details).

The general architecture of the controller is illustrated in Fig.2. As the injector is not located directly inside the cylinder, it is necessary to take into account the well-known wall-wetting phenomenon (see [2] for a mean-value model description). This phenomenon results from the fact that the injected fuel under liquid form is not instantaneously vaporized in the intake manifold: a part $X$ of the injected quantity constitutes a liquid fuel film on the manifold walls. The injected mass fuel $M_{i n j}$ and the fuel mass admitted in the cylinder $M_{f u e l}$ are related by a static relation, noted $T_{(\tau, X)}$ in Fig.2.

To validate the proposed strategy, we consider an increasing torque variation at constant engine speed (1000 rpm), followed by a sudden decrease.

Further, to test our controller under real representative driving conditions, experiments are conducted on a challenging part of the new European driving cycle (NEDC): 

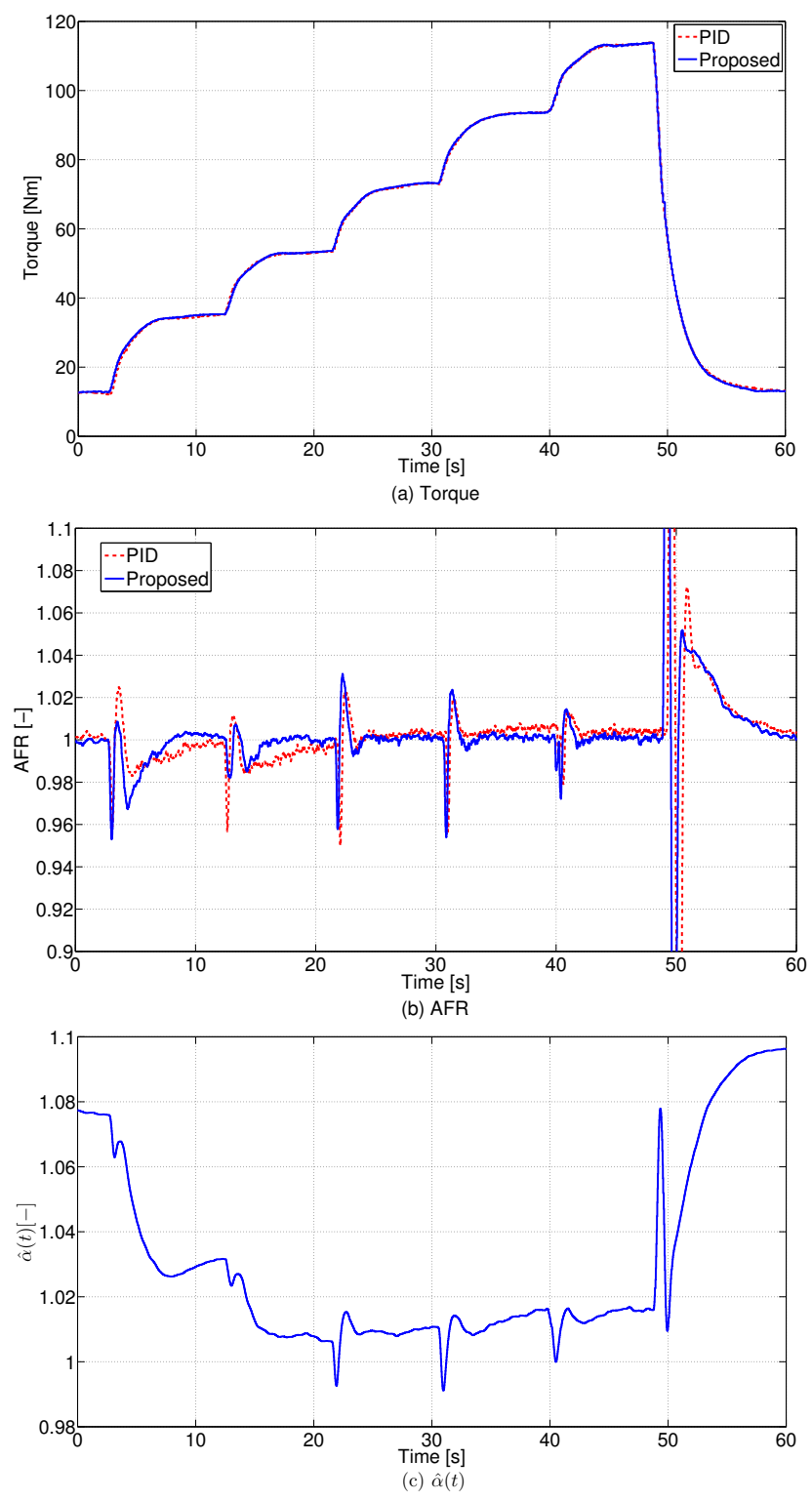

Fig. 3. Test-bench results for a constant engine speed of $1000 \mathrm{rpm}$, for the torque demand (a) : with the proposed strategy (blue) and a tuned PID (red).

it consists in one urban driving cycle (ECE) followed by an extra-urban driving cycle (EUDC).

\section{B. Experimental results}

1) Torque trajectory at constant speed: Fig.3 reports the experimental results obtained on the presented test bench for the torque trajectory of Fig.3-(a).

Comparing the performance of the controller to a reference PID on Fig.3-(b), one can observe that the time response of the proposed controller is shorter for the two first steps of torque (2-12s and 12-22s). Besides, on the interval 30-50s, it is particularly noticeable that the convergence about the value $\phi^{r}=1$ is tighter. These results highlight the benefits of our strategy, which does not require any fine gains tuning for each operating point, unlike the considered PID.

Fig.3-(c) shows the histories of the estimator $\hat{\alpha}(t)$ throughout this experiment. Its behavior is well explained by (34).
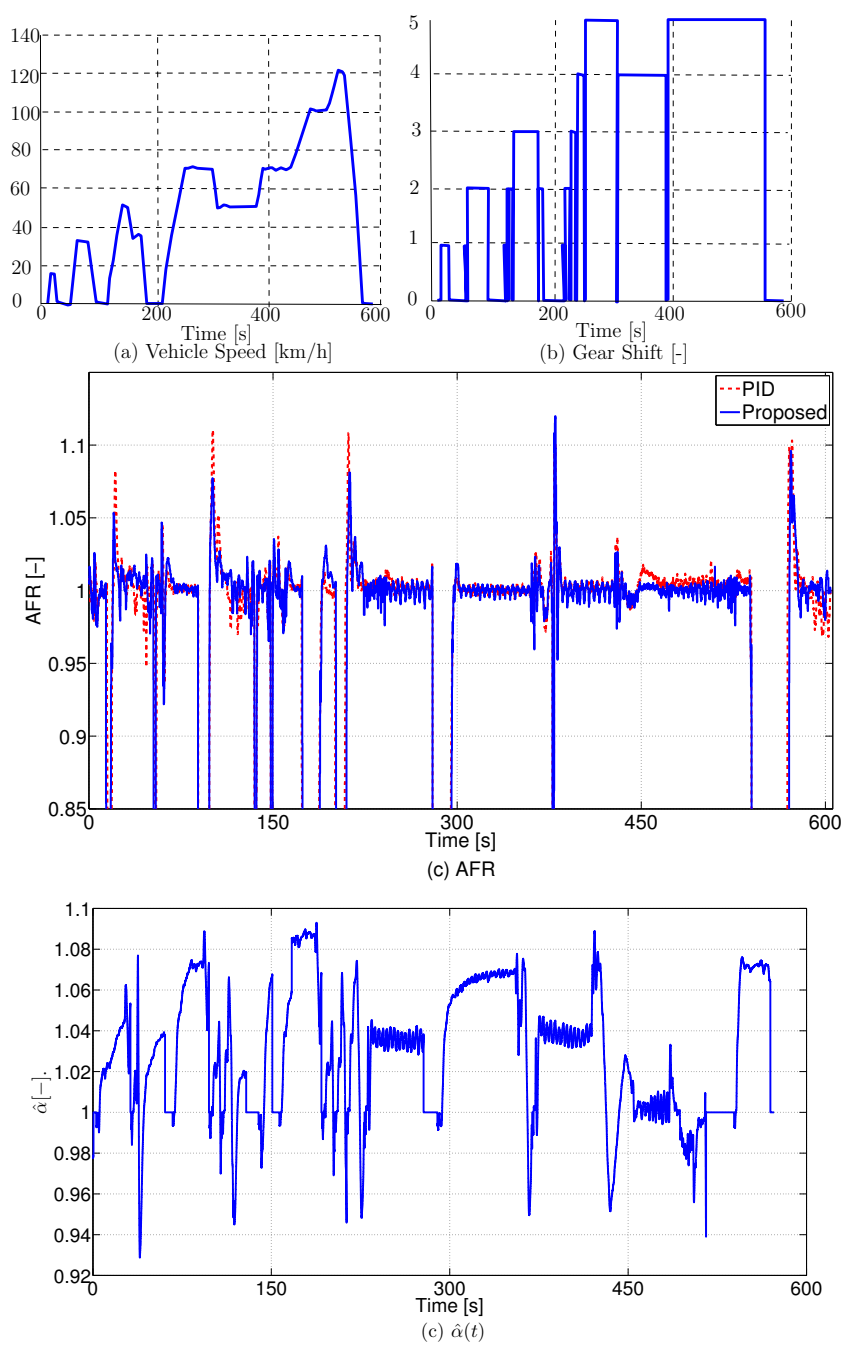

Fig. 4. Test bench results during a normalized ECE (0-200 s) cyle and an EUDC (200-600 s) cycle : with the proposed strategy (blue) and a tuned PID (red).

In details, when the convergence of the AFR and the control have been obtained, the estimate error $\tilde{\alpha}(t)$ is zero, which means that the estimate parameter $\hat{\alpha}(t)$ has converged to the unknown value $\alpha$. This result, which unfortunately cannot be generalized to multi-parameters estimation (as is well known in adaptive control [9]) is of great interest in the context of engine diagnosis.

2) NEDC cycle: generally speaking, this demanding test yields similar conclusions. Results are reported in Fig.4. A tight convergence is obtained with the proposed strategy, particularly for a gear shift above 3 (corresponding to the time interval 250-600s). More precisely, this test stresses the relevance of the proposed controller over a large range of operating points and under real driving conditions (injection shut-off corresponding to the sudden drops of AFR in Fig.4(c)). Finally, Table I quantatively summarizes the benefits of the proposed strategy on the two previous tests.

\section{CONCLUSiOns AND Future WORKS}

This paper presents a general adaptive control strategy for systems with unknown input time delay and unknown parameters in the plant. The proposed controller has been 


\begin{tabular}{|c|c|c|}
\hline Test & Constant Speed & NEDC \\
\hline PID performances & 0.0541 & 0.1622 \\
\hline Adaptive control performances & 0.0464 & 0.1286 \\
\hline $\begin{array}{c}\text { Relative gain } \\
\text { compared with PID }\end{array}$ & $14 \%$ & $20 \%$ \\
\hline
\end{tabular}

TABLE I

COMPARISON BETWEEN THE PERFORMANCES OF THE TWO CONTROLLERS, MEASURED By $\int_{\{t}$ : INJECTION ON $\} \tilde{\phi}(t)^{2} d t$.

applied with success to the regulation of the Air-Fuel Ratio in Spark Ignition engines.

The complete proof in the general multi-dimensional case will be the subject of upcoming work. Nevertheless, several other points must also be explored. As was highlighted, no particular effort had to be made on the delay estimate, which was modeled by a constant sufficiently close to the real value. The design of an adaptation law for the delay estimate seems a good idea to improve the transient performance of the controller. This point will be a key to track a non-constant trajectory.

\section{APPENDIX}

\section{A. Expression of $f$ and $g$}

Using the fact that $\int_{0}^{x} \int_{0}^{y} \psi(x, y, \xi) d \xi d y=$ $\int_{0}^{x} \int_{\xi}^{y} \psi(x, y, \xi) d y d \xi$, jointly with the transformation (33) and its inverse (43), one can obtain

$$
\begin{aligned}
& f(x, t)=\frac{\hat{w}_{x}(x, t)}{\hat{D}}-\frac{\hat{\alpha}(t)}{\tau_{\phi}} \hat{w}(x, t)+\frac{1+\hat{\alpha}(t)}{\tau_{\phi}}\left(e^{-(1+\hat{\alpha}(t)) \hat{D} x / \tau_{\phi}}\right. \\
& \left.\times \tilde{\phi}(t)+\frac{\hat{\alpha}(t) \hat{D}}{\tau_{\phi}} \int_{0}^{x} e^{-(1+\hat{\alpha}(t)) \hat{D}(x-y) / \tau_{\phi}} \hat{w}(y, t) d y\right) \\
& g(x, t)=\frac{\hat{D}}{\tau_{\phi}}\left(\int _ { 0 } ^ { x } \hat { w } ( y , t ) \left[e^{-\hat{D}(x-y) / \tau_{\phi}}\right.\right. \\
& \left.-\hat{D} \frac{\hat{\alpha}(t)}{\tau_{\phi}} \int_{y}^{x} e^{-\hat{D}(x-\xi+(1+\hat{\alpha}(t))(\xi-y)) / \tau_{\phi}} d \xi\right] d y \\
& \left.-\tilde{\phi}(t) \int_{0}^{x} e^{-\hat{D}(x+\hat{\alpha}(t) y) / \tau_{\phi}} d y\right) \\
& +\frac{\phi^{r}}{\hat{\alpha}(t)^{2}}\left(1+\frac{\hat{D}}{\tau_{\phi}} \int_{0}^{x} e^{-\hat{D}(x-y) / \tau_{\phi}} d y\right)
\end{aligned}
$$

\section{B. Bounds of the terms involved in the Lyapunov Analysis}

Using Cauchy-Schwartz inequality, Young's inequality and Agmon's inequality $\hat{w}(0, t)^{2} \leq 4\left\|\hat{w}_{x}(t)\right\|^{2}$ (along with the fact that $\hat{w}(1, t)=0)$, one obtains

$$
\begin{aligned}
& 2|h(t)||e(0, t)|=|h(t) \| \tilde{e}(0, t)+\hat{w}(0, t)-\tilde{\phi}(t)| \\
& \quad \leq M_{1}\left(\tilde{\phi}(t)^{2}+\tilde{e}(0, t)^{2}+\left\|\hat{w}_{x}(t)\right\|^{2}\right) \\
& \quad+M_{2}\left(\tilde{\phi}(t)^{2}+\tilde{e}(0, t)^{2}+\|\hat{w}(t)\|^{2}+\left\|\hat{w}_{x}(t)\right\|^{2}\right) \\
& \quad+M_{3}\left(\tilde{\phi}(t)^{2}+\tilde{e}(0, t)^{2}+\left\|\hat{w}_{x}(t)\right\|^{2}\right) \\
& 2 \int_{0}^{1}(1+x)|\tilde{e}(x, t) \| f(x, t)| d x \\
& \quad \leq M_{4}\left(\tilde{\phi}(t)^{2}+\|\tilde{e}(t)\|^{2}+\|\hat{w}(t)\|^{2}+\left\|\hat{w}_{x}(t)\right\|^{2}\right)
\end{aligned}
$$

$$
\begin{aligned}
& 2 \hat{D} \int_{0}^{1}(1+x)|\hat{w}(x, t)||g(x, t)| d x \\
& \leq M_{5}\left(\tilde{\phi}(t)^{2}+\|\hat{w}(t)\|^{2}+\|\hat{w}(t)\|\right) \\
& 2 \hat{D} \int_{0}^{1}(1+x)\left|\hat{w}_{x}(x, t)\right|\left|g_{x}(x, t)\right| d x \\
& \leq M_{6}\left(\tilde{\phi}(t)^{2}+\|\hat{w}(t)\|^{2}+\left\|\hat{w}_{x}(t)\right\|^{2}+\left\|\hat{w}_{x}(t)\right\|\right) \\
& 2 \hat{D} \frac{\hat{\alpha}(t)}{\tau_{\phi}}|\tilde{e}(0, t)| \int_{0}^{1}(1+x)|\hat{w}(x, t)| e^{-\hat{D} x / \tau_{\phi}} d x \\
& \leq 2 M_{7} \tilde{e}(0, t)^{2}+\|\hat{w}(t)\|^{2} / 2 \\
& 2 \frac{\hat{D}^{2} \hat{\alpha}(t)}{\tau_{\phi}^{2}}|\tilde{e}(0, t)| \int_{0}^{1}(1+x)\left|\hat{w}_{x}(x, t)\right| e^{-\hat{D} x / \tau_{\phi}} d x \\
& \leq 2 M_{8} \tilde{e}(0, t)^{2}+\left\|\hat{w}_{x}(t)\right\|^{2} / 2 \\
& 2 \hat{w}_{x}(1, t) \leq M_{9}|\dot{\hat{\alpha}}(t)|^{2}\left(\|\hat{w}(t)\|^{2}+1\right)+M_{10} \tilde{e}(0, t)^{2} \\
& +M_{11} \tilde{\alpha}(t)^{2}\left(\tilde{e}(0, t)^{2}+\tilde{\phi}(t)^{2}+\left\|\hat{w}_{x}(t)\right\|^{2}\right) \\
& |\dot{\hat{\alpha}}(t)| \leq \gamma M_{12}\left(|\tilde{\phi}|+\|\hat{w}(t)\|+\left\|\hat{w}_{x}(t)\right\|\right)
\end{aligned}
$$

with, for example, $M_{1}=\frac{4}{b_{2}}, M_{2}=\frac{8 \bar{D}}{\tau_{\phi}}, M_{3}=\frac{14 \bar{D}^{2}}{\tau_{\phi}^{2}}, \ldots$,

\section{REFERENCES}

[1] C. Alippi, C. de Russis, and V. Piuri. A neural-network based control solution to air-fuel ratio control for automotive fuel-injection systems. IEEE Transactions on Systems, Man, and Cybernetics, Part C: Applications and Reviews, 33(2):259-268, 2003.

[2] C. F. Aquino. Transient A/F control characteristics of the 5 liter central fuel injection engine. SAE International Journal of Passenger CarsElectronic and Electrical Systems, 1981.

[3] I. Arsie, C. Pianese, and M. Sorrentino. Development and real-time implementation of recurrent neural networks for AFR prediction and control. SAE International Journal of Passenger Cars-Electronic and Electrical Systems, 1(1):403, 2009.

[4] Z. Artstein. Linear systems with delayed controls: a reduction. IEEE Transactions on Automatic Control, 27(4):869-879, 1982.

[5] D. Bresch-Pietri and M Krstic. Delay-adaptive full-state predictor feedback for systems with unknown long actuator delay. IEEE Transactions on Automatic Control. submitted.

[6] D. Bresch-Pietri and M. Krstic. Adaptive trajectory tracking despite unknown input delay and plant parameters. Automatica, 45(9):20742081, 2009

[7] A. Chasse, P. Pognant-Gros, and A. Sciarretta. Online implementation of an optimal supervisory control for hybrid powertrains. SAE International, 2009.

[8] M. F. Hsieh, M. Canova, and J. Wang. Model predictive control approach for AFR control during lean NOx trap regenerations. SAE International Journal of Fuels and Lubricants, 2(1):149, 2009.

[9] P. A. Ioannou and J. Sun. Robust adaptive control. Prentice Hall Englewood Cliffs, NJ, 1996.

[10] M. Krstic. Boundary control of PDEs: A course on backstepping designs. Society for Industrial and Applied Mathematics Philadelphia, PA, USA, 2008.

[11] M. Krstic and A. Smyshlyaev. Backstepping boundary control for first-order hyperbolic PDEs and application to systems with actuator and sensor delays. Systems \& Control Letters, 57(9):750-758, 2008.

[12] M. Locatelli, E. Alfieri, C. H. Onder, and H. P. Geering. Identification of the relevant parameters of the wall-wetting system by extended Kalman filtering. Control Engineering Practice, 14(3):235-241, 2006.

[13] Z. Palmor. Stability properties of Smith dead-time compensator controllers. International Journal of Control, 32:937-49, 1980.

[14] J.-P. Richard. Time-delay systems: an overview of some recent advances and open problems. Automatica, 39(10):1667-1694, 2003.

[15] D. Y. Wang and E. Detwiler. Exhaust oxygen sensor dynamic study. Sensors \& Actuators: B. Chemical, 120(1):200-206, 2006.

[16] Q. C. Zhong. Robust control of time-delay systems. Springer Verlag, 2006. 